\section{Dynamic value assessments in oncology supported by the PACE Continuous Innovation Indicators}

\author{
Silvia Paddock', Clifford Goodman², Scott Shortenhaus ${ }^{3}$, David Grainger ${ }^{4}$, \\ Jacqueline Zummo Z Samuel Thomas $^{*, 1}$
}

\begin{abstract}
Aim: Several recently developed frameworks aim to assess the value of cancer treatments, but the most appropriate metrics remain uncertain. Methods: We use data from the Patient Access to Cancer care Excellence Continuous Innovation Indicators to examine the relationship between hazard ratios (HRs) from clinical trials and dynamic therapeutic value accumulating over time. Results: Our analysis shows that HRs from initial clinical trials poorly predict the eventual therapeutic value of cancer treatments. Conclusion: Relying strongly on HRs from registration trials to predict the long-term success of treatments leaves a lot of the variance unexplained. The Continuous Innovation Indicators offer a complementing, dynamic method to track the therapeutic value of cancer treatments and continuously update value assessments as additional evidence accumulates.
\end{abstract}

First draft submitted: 24 May 2017; Accepted for publication: 4 July 2017;

Published online: 26 July 2017

Concerns about increased healthcare costs and the ability to pay for meaningful health improvements have fueled discussions about value in healthcare. Various organizations have responded by developing value frameworks to assess the relative benefits of therapeutic interventions. These frameworks typically assess new treatments at the time of launch based on data from initial registration trials and other available information. Some of these frameworks rely mainly on hazard ratios (HRs) of primary end points from registration trials, because these measures often provide the only available estimate of efficacy. However, the extent to which HRs from initial trials predict future value of new therapies is unclear. Moreover, while current value frameworks provide a good initial basis for conceptualizing value, they are not designed to consider value across the lifecycle of a treatment, and they inadequately account for patient preferences.

Several examples of published case studies attempt to quantify value dynamically. Among these is the application of Garrison and Veenstra's dynamic lifecycle model to trastuzumab to calculate annual incremental cost-effectiveness ratios (ICERs) for two indications: metastatic breast cancer and adjuvant treatment for early breast cancer [1]. That model demonstrated a decrease over time in ICERs for the combined indications. Similarly, Lu et al. conducted an analysis of the dynamic cost-effectiveness of docetaxel and paclitaxel as new indications arose and patents expired [2]. They concluded, "One direction for future research might be to create a predictive tool that estimates the predicted long-term dynamic ICER for a specific drug at product launch. ... All these sources of information can be used together to predict the probability of different events over a drug's life cycle."

'Rose Li \& Associates, Inc., 9501 Wadsworth Dr., Bethesda, MD 20817, USA

${ }^{2}$ Center for Comparative Effectiveness Research, The Lewin Group, 3130 Fairview Park Dr., \#500, Falls Church, VA 22042, USA

${ }^{3}$ Eli Lilly and Company, Lilly Corporate Center, Indianapolis, IN 46285, USA

${ }^{4}$ Eli Lilly and Company, 112 Wharf Rd., Melrose Park, NSW 2114, Australia

*Author for correspondence: +1 202549 4388; samuel.thomas@roseliassociates.com

\section{KEYWORDS}

hazard ratio $\bullet$ health economics $\bullet$ value assessment 
We herein offer one tool to support such dynamic assessments: the PACE Continuous Innovation Indicators (CII), a publicly accessible policy tool to track and quantify evidence for increased survival achieved by treatments for 12 solid cancer types. To date, we have screened more than 12,000 publications and analyzed and curated pieces of evidence from approximately 3000 abstracts and full text articles. The CII allows for dynamic adjustments of therapeutic value - defined as published evidence for improved overall survival (OS) - based on specific indications. Based on scientific evidence rather than clinical guidelines, the CII is not meant to directly inform individual treatment decisions or be used by patients to guide their own care. It is intended to support multiple stakeholders - including physicians, patient advocates, health services researchers, health economists, payer representatives and others who seek to determine the value of oncology treatments, track progress, identify unmet needs, assess new health technologies and shape healthcare policies and systems. The CII also does not include any information on cost, although it can be combined with data from other sources to derive estimates of 'value for money'.

We present several examples of progress to date against cancer. We then show that measures proposed to serve as indicators of clinical value that is, HRs for OS reported from clinical trials - are poor predictors of long-term gains. Finally, we offer several examples of how CII data can support dynamic assessments of the value of new and continuing interventions in oncology.

\section{Methods}

The detailed methodology underlying the PACE CII data capture and visualization process has been published previously [3]. The tool is freely available online [4].

The PACE Dynamic Value Matrix (Figure 1) is a visual framework that enables users to assign weights to different treatment goals and to obtain a quantitative measure of progress. Each circle represents a unique treatment, and all treatments are classified by the disease state and treatment goal to which they are applied.

We include all treatments that have shown evidence for increased OS in at least one published study (Figure 2A). We also include information about regulatory approval dates in our database, which allows us to track new evidence in relation to market access. As such, treatments that are approved based on surrogate end points and later show evidence of survival are represented in the database. If a treatment shows evidence for increased survival in different disease states or for different treatment goals over time, it occurs in multiple rows of the database and is plotted multiple times on the matrix.

The CII incorporates abstracted data from published literature, including Phase I and III clinical trials, observational studies, systematic reviews and meta-analyses. Each reference is parsed into one or more 'pieces of evidence' that reflects a single comparison of OS outcomes between two treatment arms. Thus, a three-arm clinical trial would produce at least two pieces of evidence. Each piece of evidence contains the experimental treatment name, OS statistics, treatment context (defined by the PACE Dynamic Value Matrix), subgroup requirements and other relevant information.

Figure 2B shows how the PACE CII calculates the Evidence Score (E-Score), a quantitative measure of progress over time that is a function of treatment goal (indication) achieved for a given disease state; "pieces of evidence" (i.e., findings for a particular health outcome) in support of each treatment goal achieved; and strength of each piece of evidence (based on trial or other study design). That is, an E-Score is the sum of the number of pieces of evidence supporting a treatment times the weight assigned to each type of study (e.g., randomized controlled trial, meta-analysis) that generated each piece of evidence times the weight assigned to each indication achieved by that treatment (matrix squares). On the interactive website, users can assign their own value weights to the different squares on the PACE Dynamic Value Matrix. Although the CII systematically records information about effect size, these measures are not factored into the E-Score algorithm, because they are not always comparable across the different designs of studies within the database.

The database also captures negative study results, although they generally have no effect on E-Scores. This is because negative results do not represent negative progress - in fact, negative results often provide important scientific lessons that advance future research. Nonetheless, it is important that false-positive data do not inflate E-Scores. Thus, if a study is refuted by later evidence, such as a pivotal Phase III trial or metaanalysis, the CII excludes the earlier evidence in all analyses from E-Score calculations without 


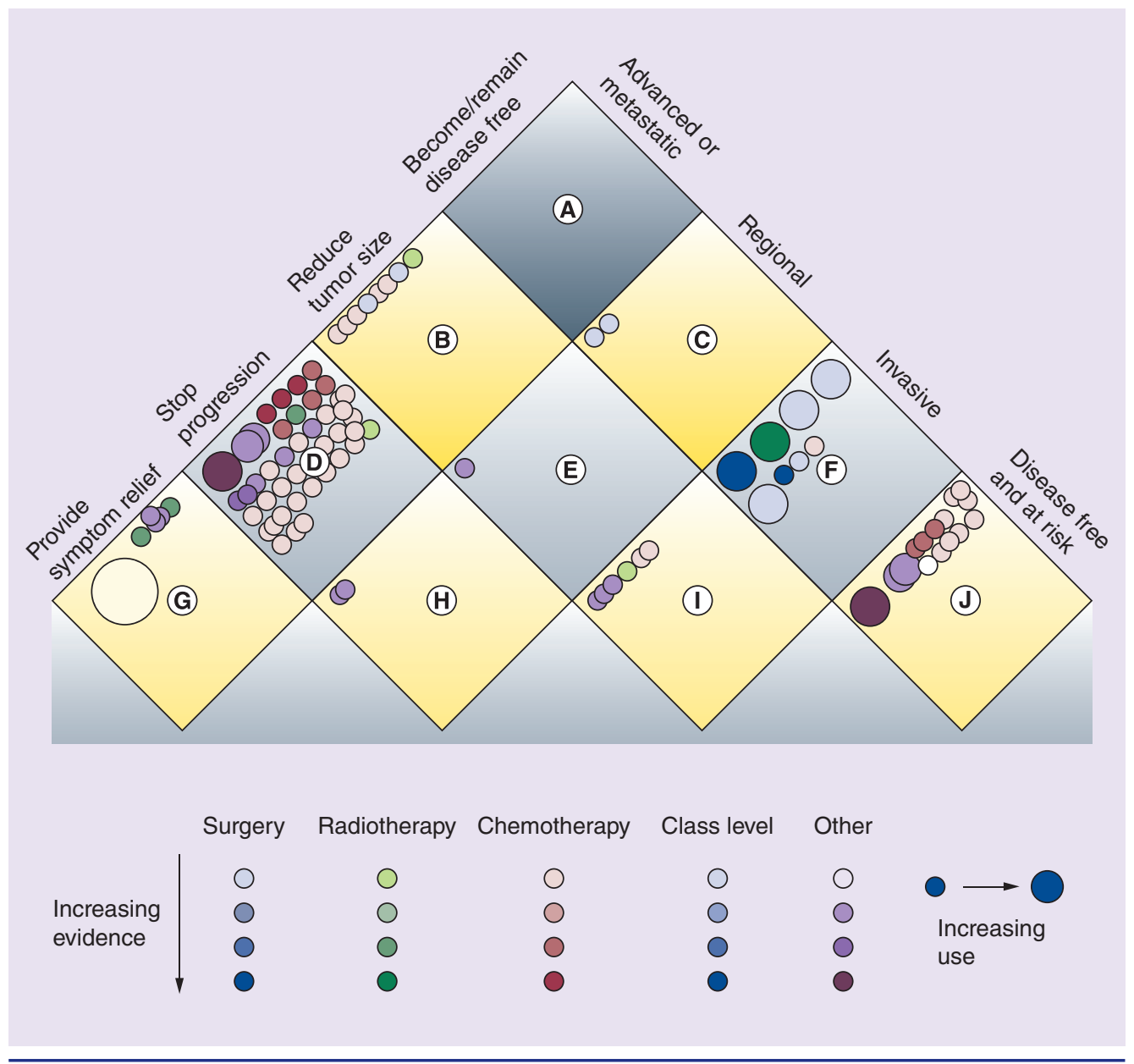

Figure 1. The Patient Access to Cancer care Excellence Dynamic Value Matrix. Each circle represents a unique treatment. Treatments are classified by the disease state (right axis) and treatment goal (left axis) to which they are applied. Color indicates the treatment modality. Color intensity indicates the strength of the evidence supporting the treatment, where darker circles represent treatments supported by a greater number of pieces of evidence. Combination treatments are shown as clusters of circles. The size of circles denotes whether the treatment is currently offered to only a few patients (small), to most patients (medium) or to essentially all patients (large) with a given indication.

deleting the initial record from the database. The earlier evidence can be revisited later in the context of new evidence. This mechanism allows for systematic tracking of evidence as it emerges while ensuring that false-positive results do not inflate E-Scores.

Statistical analyses were performed using $\mathrm{R}$ (version 3.3.1) by fitting linear models to dates, E-Scores and HRs downloaded from the public PACE CII database after eliminating one outlier (defined as lying more than four standard deviations above the mean). To study the effect of HRs on E-Scores while accounting for the effect of the publication year, we first fitted a linear model to capture the effect of time on the E-Scores and then fitted a second linear model to the residuals of the time E-Score linear model, that is, the unexplained part of the variance. We performed these analyses both for the first HRs reported for each treatment and for the best HRs, as detailed in the results below.

All data used in this study have been downloaded from the PACE CII public database, a resource sponsored by Lilly Oncology. Charts used in the dynamic value figures have been generated on the PACE CII 'Approval Date Analysis' tab. 


\section{Results}

- Most evidence for increased OS is obtained after approval

Most treatments that are current standards of care in cancer have been substantiated with evidence that accumulated after their initial regulatory approval. A review of evidence of all systemic treatments across the database shows that most of the bodies of evidence for increased OS were obtained after the treatments were introduced into clinical practice (Figure 3 ).
The peri-approval period in Figure 3 spans from 2 years prior to the approval year until 2 years after the approval year to account for possible delays in publishing evidence in conjunction with the approval process. Pre-approval and postapproval evidence is gathered before and after that period. The chart on the left shows that many treatments gain evidence for increased OS after the approval. The chart on the right shows that this effect is most prominent for breast, colorectal and non-small-cell lung cancers.
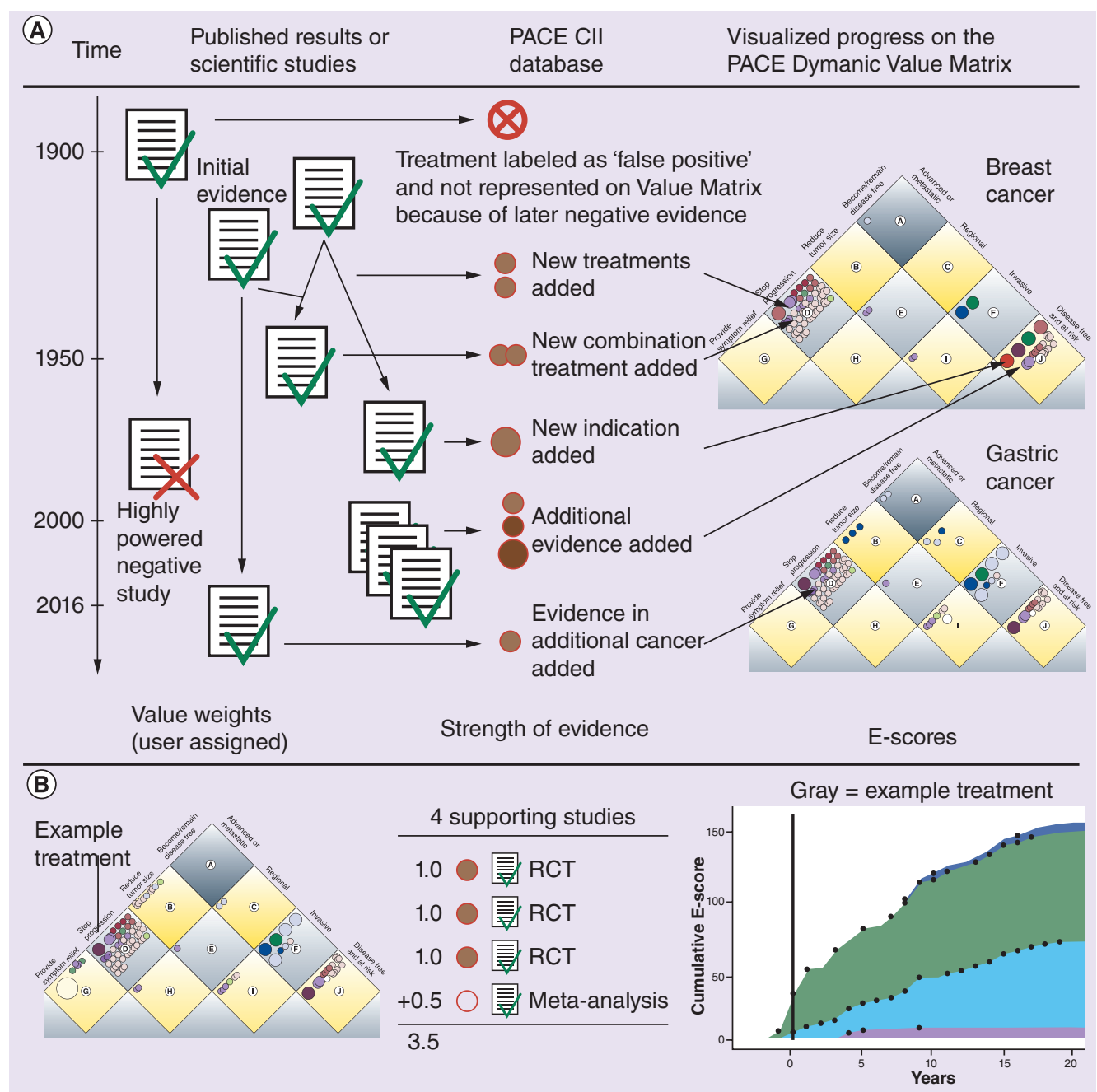

\begin{tabular}{lccccc|}
\hline Formula & Value weight & & Evidence weight & Evidence score \\
example: & 2 & 3.5 & 7 \\
\hline
\end{tabular}

Figure 2. Schematic of Evidence Score Calculation. (A) Depicts the Cll process for selecting evidence for inclusion on the PACE Dynamic Value Matrix and in E-Score calculations. Green check marks indicate studies with statistically significant evidence for overall survival in a preplanned analysis. (B) Provides the E-Score calculation algorithm and an illustrative example. CII: Continuous Innovation Indicator; E-Score: Evidence Score; PACE: Patient Access to Cancer care Excellence. 


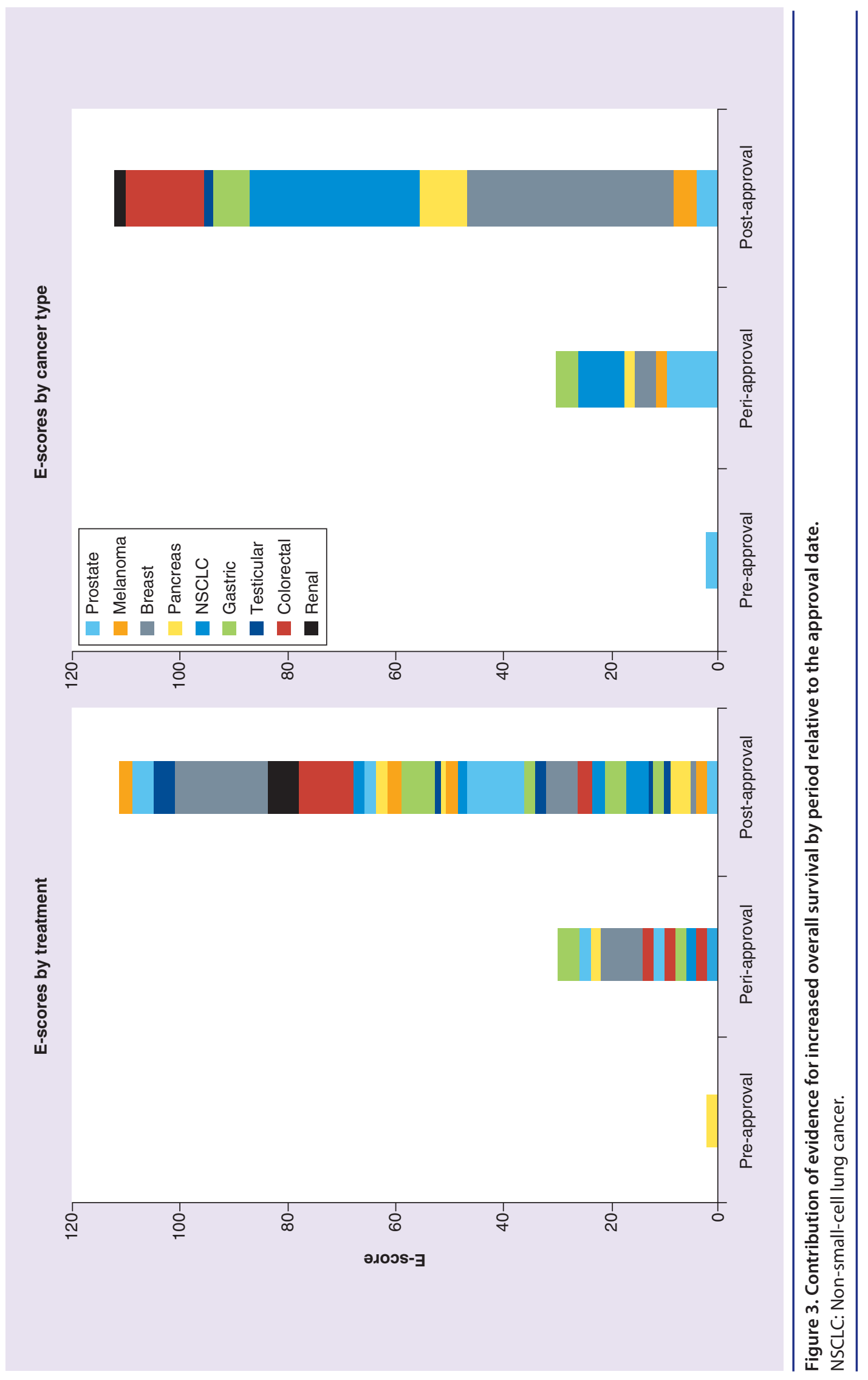




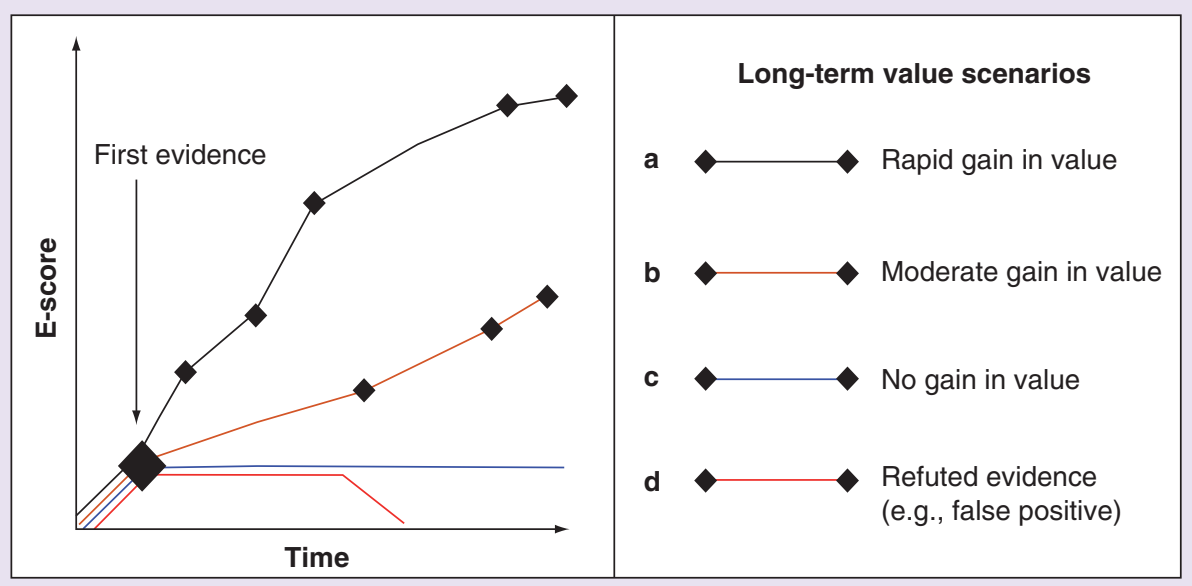

Figure 4. Value path examples. Treatment (A) gains substantial additional value after approval. Treatment (B) accumulates evidence more slowly. Treatment (C) remains at a static level. Initial evidence for treatment (D) is later refuted.

The columns on the left show a substantial variability between different treatments: some treatments (e.g., yellow blocks) show little additional evidence over time, whereas others (e.g., brown blocks) continue to accumulate substantial amounts of evidence. To assess value dynamically, we would want to be able to predict which treatments continue to be successful and increase in value, and which do not.

Figure 4 illustrates this challenge schematically. At the time of approval (first black milestone), we would like to know how the treatment will fare over time. Will it continue to grow in value rapidly (Figure 4A), more slowly and with greater intervals between new pieces of evidence (Figure 4B) or not at all (Figure 4C), or will existing evidence later be refuted (Figure 4D)? Current, static value frameworks essentially treat all new treatments as if they followed path (Figure 4C): the assessment is solely based on the first evidence at approval without accounting for future potential increases in value.

- HRs from clinical trials poorly predict the dynamic evolution of therapeutic value How well do HRs predict a treatment's total clinical value over time? Results from linear model fitting analyses are shown in Figure 5. Figure 5A shows that there is no correlation between the first HRs retrieved from the database and the ultimate E-Scores as measures of dynamic, cumulative therapeutic value. There is a trend toward a weak correlation in Figure 5B, when we select the best HR for each treatment (instead of the first one). As one would expect, there is a highly significant correlation between the time of the first reported HR in the database and the total E-Score, simply because longer-known treatments had more chances to increase their score (Figure $5 \mathrm{C}$ ). We can adjust for this effect and re-test the hypotheses from Figures 5A \& 5B after accounting for time. Results from the analysis of the first HR reached significance $\left(\mathrm{p}<0.01 ; \mathrm{R}^{2}=0.09\right)$, and results from the best-ever $\mathrm{HR}$ analysis also reached significance $(\mathrm{p}<0.01$; Figure $5 \mathrm{C})$, albeit with an $\mathrm{R}^{2}$ of 0.07 , indicating only a weak correlation. It is, therefore, not possible to know from the HR of OS in the first trial alone which of the treatments will eventually be supported by a robust body of published evidence.

\section{- What other measures can assist to predict future value?}

Value assessment should be dynamic, based on the recognition that ongoing drug development and evidence generation reduce uncertainty. The CII can help to develop such dynamic value estimates.

As is evident from Figure 6 portraying cumulative E-Scores of five treatment classes over time, a static value assessment derived from the first trial of any of these treatment classes (red ellipse) showing evidence for efficacy in OS would far under-represent the impact of the treatment as demonstrated in subsequent trials. Green triangles indicate dynamic value assessments that account for the accumulation of evidence over time. 


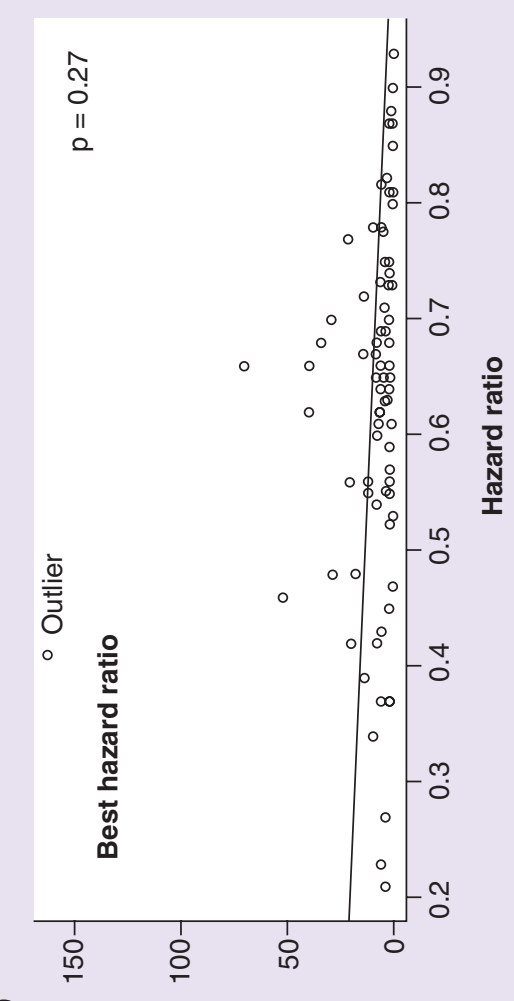

(๓)

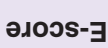

o
0
0
II
0

○ั

$\varangle$

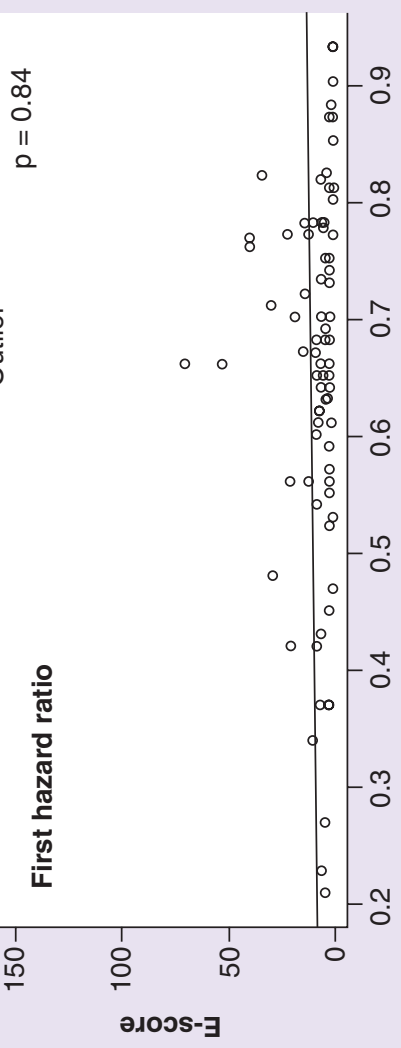

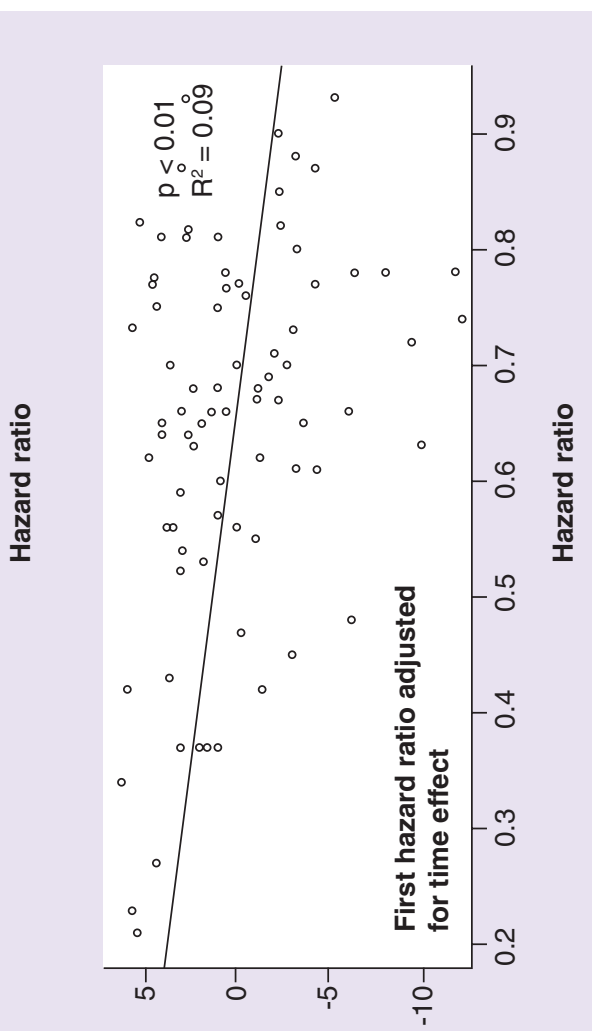

(2)
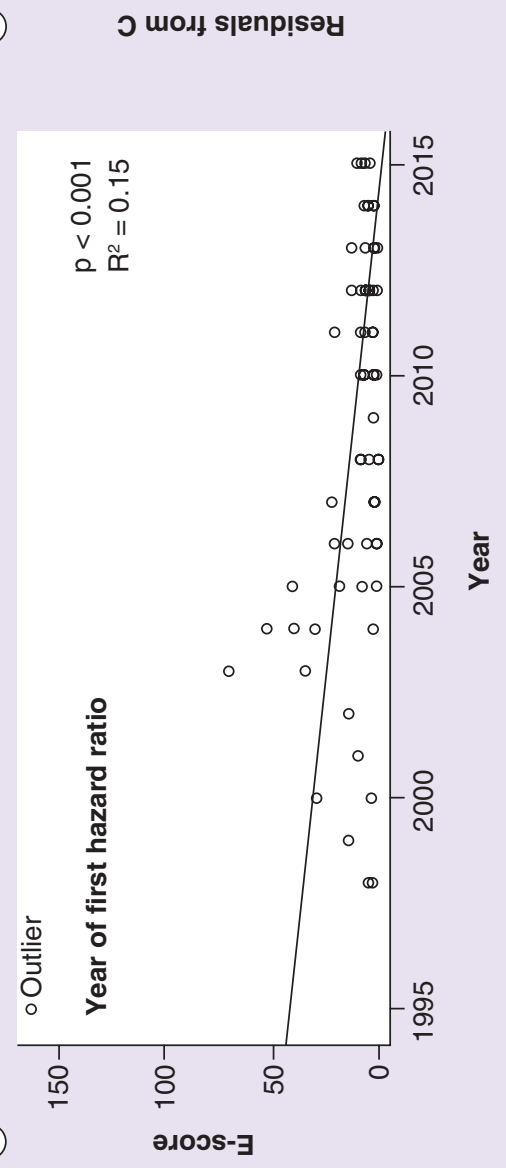

ᄃ

过 㫕 है ज恼 䍃苛 全突

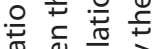
흔 岂 웡

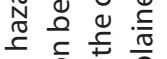
응 음 苟 ๙ 항 苋艺施 记

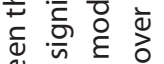

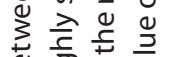

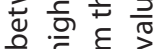
ᄃ

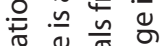

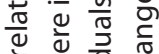
흔 흔

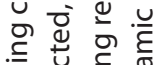

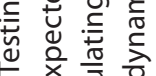
¿

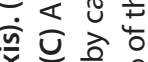
ํㅜㅇ 츄워

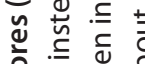
은 ज䜦

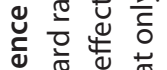
웡 豈疍它

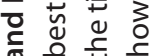

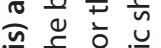
希 尔 产. 全

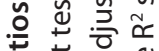

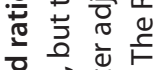

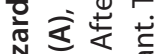

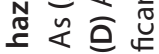

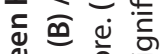
$\sum_{0}^{0} \dot{0} \overline{0}$ 茪它出 o तै 을

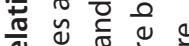

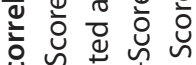

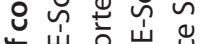
넝

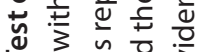

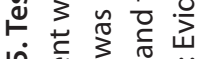
ம்

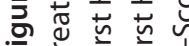


A review of the evidence accumulation since the time of approval shows that different classes of treatment experience different patterns of evidence accumulation. Monoclonal antibodies, which are a relatively recent addition to cancer care, are usually first tested in the palliative setting in patients with prognoses for short survival. As a group, monoclonal antibodies have accumulated evidence faster than any other treatment class in the database. Thus, when new, similar, treatments enter clinical practice, we can anticipate that their E-Scores - and potential value to clinicians, patients and others - could change in clinically meaningful ways over time, and that their evidence milestones might evolve accordingly.

Taxanes, another important class of therapies for many solid tumors, have accumulated substantial evidence for OS. However, in part because these treatments were initially approved based on surrogate outcomes and are often used in the adjuvant context, it has taken many years since their approval to accumulate the evidence (blue arrow in Figure 6) used today to inform clinical decisions and policies. When determining the value of similar treatments (e.g., adjuvant agents for earlystage breast cancer), we might expect similar delays in the accumulation of evidence and should consider setting our expectations and milestones accordingly.

Although platinum compounds are a longstanding class of therapies in oncology, they continue to accumulate clinically relevant evidence. This includes an expanding set of cancer care indications. Decision makers should consider how value can change along similarly long timelines of evidence development for potentially multiple indications. Such benefits can extend beyond, or arise after, market exclusivity periods.

Pyrimidine analogs have shown recent evidence for improvement in survival, more than three decades after their initial approval. A newer class, aromatase inhibitors, has had less time to accumulate evidence for increased OS.

The green triangles can be projected and adjusted based on the above considerations for new treatments entering the market. The slopes of the hypotenuses can inform Bayesian models that require a prior estimate of future value developments. The timing and spacing of historic evidence can be used to set milestones after which the dynamic assessment of value is updated and revised.

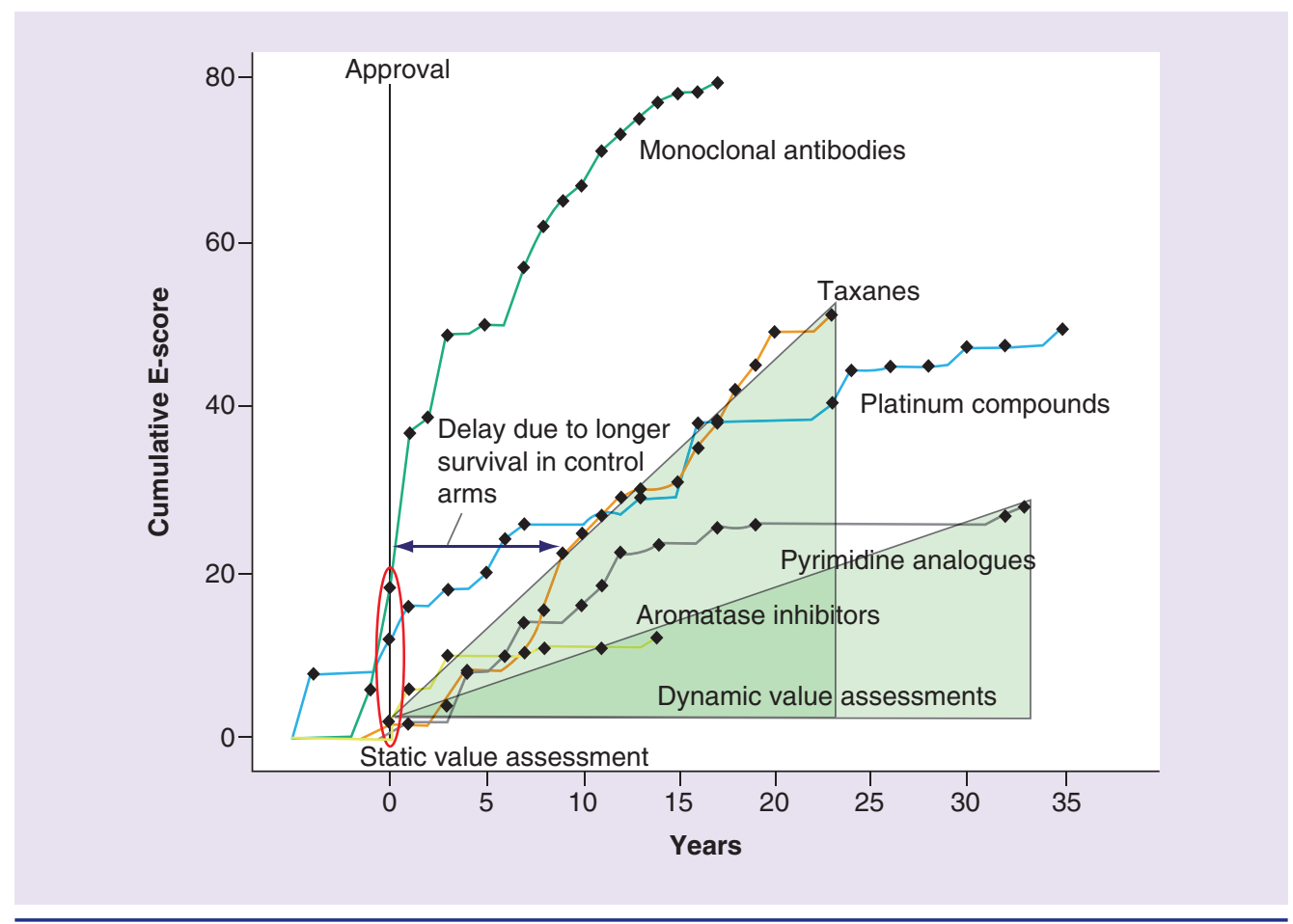

Figure 6. Dynamic value assessments with the Patient Access to Cancer care Excellence Continuous Innovation Indicators. 


\section{Discussion}

Several currently prominent value frameworks rely partly or entirely on HRs from early trials, often the registration trial for the drug [5-9]. As described above, this approach poorly predicts findings of subsequent evidence accumulation. The focus on HRs from clinical trials is further complicated by the 'efficacy-effectiveness gap', the commonly observed discrepancy between clinical trial results and the performance of a treatment in real-world practice [10]. For welldocumented reasons - including healthcare system characteristics, clinical trial measurement methods and complex interactions between a drug's biological effect and contextual factors - clinical trials do not always predict realworld outcomes [11]. Results obtained in formal trials can over- or underestimate real-world effectiveness.

Of course, HRs are attractive because they are readily available for statistical modeling. Here we show how alternative sources of data can be added to develop experience-based value models. The extensive expansion over the past three decades in the evidence base for cancer therapeutics enables a systematic approach to apply this evidence to inform future value estimates.

\section{- Dynamic value assessments based on the CII}

The Institute for Clinical and Economic Review acknowledged the importance of contextual considerations and '[other] benefits or disadvantages' of treatments when assessing their value. Recently, Institute for Clinical and Economic Review proposed use of a voting procedure to score a specified set of benefits or disadvantages and consideration of context to adjust a threshold ICER for a single value-based price benchmark [12]. The CII also provides relevant quantitative measures, enabling insights that may not be captured by a cost-effectiveness analysis conducted at a given point in time. The CII can be used in various ways to anticipate or project potential value improvements and to dynamically assess value of new treatments. For example:

- Inspect the PACE Dynamic Value Matrix entry square for a new treatment. What other treatments are there? How long did it take for those treatments to produce OS evidence? Calculate a median year to expect OS data.

- Assess the unmet needs. Are treatments available in the 'A' square of the respective matrix?
Are other needs unmet (e.g., adjuvant [square 'J' on the matrix])? Adjust projected value estimates based on unmet needs.

- Calculate the likelihood (based on prior data of treatments with similar characteristics; the slope of the E-Score can be adapted for this purpose) that the new treatment, alone or in combination with other treatments, will show value in additional squares. How might the new treatment fit into treatment goal paths that connect several elements across the matrix? Adjust the estimate based on the likelihood of these events based on historical comparisons as shown in Figure 6.

Based on the results above, determine an initial value estimate and set milestones, after which this assessment can be updated with new evidence. Patent and market exclusivity periods would also need to be considered during this process, because the introduction of generic competition can change the course of evidence generation and often results in lower prices, which may affect assessments of overall value.

\section{- Limitations of our method}

In this analysis, we use E-Scores from the PACE CII as proxies for cumulative therapeutic value. These scores are based in part on numeric weights assigned to the PACE Dynamic Value Matrix squares by an expert panel. Others may assign different values, and therefore, our online tool allows users to explore the possible effects of differing weighting schemes.

Our model further assumes that users are willing to accept trade-offs between the value of treatments responding to urgent unmet needs and the strength of supporting evidence. For example, a treatment on a 'lower' matrix position must provide stronger evidence to obtain the same E-score as a treatment placed in a higher square. Whether such trade-offs are adequate or necessary is a matter of debate in the field [13]. These considerations are very complex, and often there may not be a 'correct' answer but different answers based on cultural, personal and other contextual differences. We do not try to eliminate these differences but provide a platform for users to assess the effects of such differences on value assessments.

Our tool does not include calculations of cost but is meant to assist users to better define the numerator in the frequently cited equation of Value $=$ Outcomes $/$ Cost by better understanding 
the dynamic development of therapeutic use of new treatments. We do not intend to replace, but complement existing frameworks with our data.

Another important limitation of our tool is that it includes only evidence of improvements in OS. Other factors, such as quality of life, are clearly important in defining therapeutic value. The CII may include such measures in the future, and value assessments should draw on additional sources of information in order to comprehensively assess the clinical value of treatments.

\section{- Patient voice is critical}

Any value assessment tool must consider foremost the outcomes that matter to patients. The current CII tool is based on scientific evidence only and is not intended to guide or inform clinical decisions.
However, this Value Matrix does offer certain dynamic advantages that could be used to complement other frameworks. The PACE Dynamic Value Matrix could, therefore, be adapted for patient-provider use and could become useful in discussing treatment options with patients, for example to answer the following:

- When the patient begins a treatment regimen, where is it located on the matrix?

- What treatments are available? What is the goal of each treatment?

- How confident are we that the treatment works?

- How does each treatment compare with other

\section{SUMMARY POINTS}

\section{Value frameworks have emerged as prominent tools to evaluate cancer treatments, but their metrics remain} unvalidated

- The value of a cancer treatment is dynamic and multidimensional. It is difficult to quantify in a single summary measure applicable to all patients.

- Although different frameworks adopt various approaches to assess value, they mainly rely on hazard ratios (HRs) from initial clinical trials to determine value.

- The most appropriate value assessment methods remain uncertain.

\section{Study objectives}

- We sought to test the validity of value assessments based on HRs of overall survival from initial clinical trials.

- The Patient Access to Cancer care Excellence Continuous Innovation Indicators (CII) is a unique data resource for systematic analyses of overall survival data for hundreds of treatments across modalities and allows for tracking of progress over time.

- The CII is freely available through an interactive online interface.

- We analyzed data from the Patient Access to Cancer care Excellence CII, using evidence scores as proxies of accumulated treatment value over time, to test for correlation between HRs from clinical trial and overall treatment value.

\section{Results}

- We found that HRs of overall survival from the initial clinical trials only explain a small share of the variance in longterm value of cancer treatments.

- There was a weak correlation between HRs and future value, indicating that the most valuable treatments tend to show extraordinary effect sizes at some point during their lifetime.

\section{Conclusion}

- Our finding that HRs from clinical trials poorly predict the long-term, dynamic therapeutic value of cancer treatments challenges current efforts to summarize value using static, standardized rubrics.

- The CII provides a supplementary, evidence-based, dynamic approach to assess the value of new and existing treatments, project their potential future value, and continuously update value estimates as additional evidence accumulates.

- We encourage researchers to use the CII database to support their own analyses, including cost-effectiveness models and economic analyses of cancer care. 
treatments in the same context?

- What other square might the patient be able to reach by undergoing a certain treatment (e.g., a tumor becomes operable after neoadjuvant treatment, which could mean a transition from square ' $\mathrm{B}$ ' to square ' $\mathrm{C}$ ' or ' $\mathrm{F}$ ')? If so, could other treatments work after the current approach?

Furthermore, systematic collection of patient-reported experiences could represent another data input for dynamic value models.

\section{Conclusion}

Most progress against cancer occurs incrementally. Today, various decision makers are exploring the use of value frameworks that assess new treatments at the time of their launch, assigning value primarily based on HRs from clinical trials conducted for registration. Our analyses demonstrate that HRs - especially those from initial trials - are poor predictors of future value. Relying too heavily on HRs may provide a misleading picture of current and future value, resulting in suboptimal allocation of resources and, ultimately, suboptimal patient outcomes. Alternative approaches are needed to more accurately predict the value of cancer treatments now and in the future.

Data from the CII are freely available to support a range of activities from educational programs for policy makers and advocates to mathematical modeling and dynamic value assessments. We encourage readers to engage with the full app online [4], and to explore the effects of different weights on the dynamic assessment of therapeutic value. Upon requests, the authors can make available additional data (e.g., all negative studies assessed in the process).

Financial \& competing interests disclosure

$S$ Shortenhaus, D Grainger, J Zummo are employees of and own stock in Eli Lilly and Company. As a full-time salaried employee of The Lewin Group, C Goodman has worked on projects/contracts with health product (including drugs, biologics, devices) companies. All payments/ honoraria for such work are paid to The Lewin Group. This study and the development of the PACE CII database was funded by Eli Lilly and Company. For their time and effort, all authors received remuneration from Eli Lilly. The authors have no other relevant affiliations or financial involvement with any organization or entity with a financial interest in or financial conflict with the subject matter or materials discussed in the manuscript apart from those disclosed.

No writing assistance was utilized in the production of this manuscript.

\section{Open access}

This work is licensed under the AttributionNonCommercial-NoDerivatives 4.0 Unported License. To view a copy of this license, visit http://creativecommons.org/ licenses/by-nc-nd/4.0/

\section{References}

Papers of special note have been highlighted as:

- of interest; $\bullet$ of considerable interest

1 Garrison LP, Veenstra DL. The economic value of innovative treatments over the product life cycle: the case of targeted trastuzumab therapy for breast cancer. Value Health 12(8), 1118-1123 (2009).

2 Lu Y, Penrod JR, Sood N, Woodby S, Philipson T. Dynamic cost-effectiveness of oncology drugs. Am. J. Manag. Care. 18(11 Suppl), S249-S256 (2012).

3 Paddock S, Brum L, Sorrow K et al. PACE Continuous Innovation Indicators - a novel tool to measure progress in cancer treatments. Ecancermedicalscience 9, 498 (2015).

- Contains full details of the Continuous Innovation Indicators methodology.

4 PACE Continuous Innovation Indicators. Rose Li and Associates, Inc., MD, USA (2017). http://scoringprogress.com
5 Schnipper LE, Davidson NE, Wollins DS et al. Updating the American Society of Clinical Oncology Value Framework: revisions and reflections in response to comments received. J. Clin. Oncol. 34(24), 2925-2934 (2016).

-• The latest version of the American Society for Clinical Oncology value framework, which relies on hazard ratios as the main measure of value.

6 Cherny NI, Sullivan R, Dafni U et al. A standardised, generic, validated approach to stratify the magnitude of clinical benefit that can be anticipated from anti-cancer therapies: the European Society for Medical Oncology Magnitude of Clinical Benefit Scale (ESMO-MCBS). Ann. Oncol. 26(8), 1547-1573 (2015).

-• Details the European Society of Medical Oncology value framework, which relies on hazard ratios in calculating value.
7 National Comprehensive Cancer Network (NCCN) Clinical Practice Guidelines in Oncology with NCCN Evidence Blocks. National Comprehensive Cancer Network, PA, USA. https://www.nccn.org/evidenceblocks/

-• The National Comprehensive Cancer Network value assessment framework presents results, but the methodology is not fully transparent.

8 ICER Value Assessment Framework. Institute for Clinical and Economic Review, MA, USA. https://icer-review.org/methodology

-• The incremental cost-effectiveness ratio value assessment framework takes a cost-effectiveness approach to value.

9 Drug Abacus. Memorial Sloan Kettering, NY, USA (2017).

http://drugpricinglab.org/tools/drug-abacus/

-. Drug Abacus is an online tool for estimating 
RESEARCH ARTICLE Paddock, Goodman, Shortenhaus, Grainger, Zummo \& Thomas

the value of select oncology treatments based on user inputs.

10 Eichler H-G, Abadie E, Breckenridge A et al. Bridging the efficacy-effectiveness gap: a regulator's perspective on addressing variability of drug response. Nat. Rev. Drug Discov. 10(7), 495-506 (2011).

11 Nordon C, Karcher H, Groenwold RHH et al. The "Efficacy-Effectiveness Gap": historical background and current conceptualization. Value Health 19(1), 75-81 (2016).
12 Overview of the ICER value framework and proposals for an update for 2017-2018 (2017). Institute for Clinical and Economic Review, MA, USA.

http://icer-review.org/wp-content

13 Eichler H-G, Baird LG, Barker R, BloechlDaum B, Børlum-Kristensen F, Brown J et al. From adaptive licensing to adaptive pathways: delivering a flexible life-span approach to bring new drugs to patients. Clin. Pharmacol. Ther. 97(3), 234-246 (2015).
- A highly relevant work by experts on regulatory issues, health technology assessments and complex discussions of value. This paper explains the need for dynamic views of value and contains discussions of the kinds of trade-offs (e.g., urgency of unmet need vs strength of evidence) that users can explore with our platform. 